\title{
Quantum features of high energy particle incoherent scattering in crystals
}

\author{
Victor V. Tikhomirov \\ Research Institute for Nuclear Problems, Belarusian State University, Minsk, Belarus
}

(Received 1 December 2018; published 13 May 2019)

\begin{abstract}
The coherent process of particle deflection by aligned atomic strings and planes of oriented crystals is accompanied by incoherent scattering by atomic cores. While the coherent particle deflection, described by the axial or planar averaged potential, becomes more and more classical at high energies, the incoherent scattering of relativistic particles remains essentially quantum. Though the incoherent scattering of relativistic particles in crystals reminds the scattering by atoms of an amorphous medium at a large momentum transfer, at small ones the incoherent scattering process in crystals is modified by the influence of the inhomogeneity of the atom distribution in the plane normal to the crystal axis or plane. We present a theory of incoherent scattering of high energy particles in oriented crystals, which takes into consideration both its quantum nature and the impact of the atom distribution inhomogeneity. The axial case is considered as a more general example. The way to incorporate the revealed quantum features into classical trajectory simulations is also outlined.
\end{abstract}

DOI: 10.1103/PhysRevAccelBeams.22.054501

\section{INTRODUCTION}

High energy particle interaction with oriented crystals makes it possible to both observe many remarkable phenomena and apply them to develop diverse sources of $x$ and gamma radiation [1-3], to efficiently deflect high energy particle beams [4-6], to measure and even to modify elementary particle properties, such as magnetic momenta [1], and to reduce the thickness of particle detectors as well as to make the latter sensitive to both the direction and polarization [7-10]. All the pronounced effects, induced by the coherent particle interaction with an oriented crystal lattice, are described by the averaged (continuum) potential of atomic strings or planes [11,12] introduced by Lindhard, who also proved that the particle motion in the averaged potential can be treated classically at a high enough energy.

Despite the large strength of the coherent effects in crystals, all their applications are essentially limited by the incoherent scattering effects relational, but not completely similar, to the scattering process in amorphous media or randomly oriented crystals. Most severely, incoherent scattering by nuclei limits the deflection of negatively charged particles by bent crystals [13,14] as well as both the channeling [1-3] and crystal undulator [1,15-17] radiation of the same. That is why, to consider any application of the coherent effects in crystals, it is

Published by the American Physical Society under the terms of the Creative Commons Attribution 4.0 International license. Further distribution of this work must maintain attribution to the author(s) and the published article's title, journal citation, and DOI. mandatory to understand and properly treat the incoherent ones. However, neither a consistent theory nor a commonly recognized view on the nature of high energy particle incoherent scattering still exist.

Channeling effect study began from the classical particle motion simulations by the binary collision method [18]. Being correct for $\mathrm{MeV}$-energy ions, the latter is inapplicable in the relativistic case, as, following Refs. [11,19-21], we recall in Sec. II. This way, a fundamental problem of treating quantum effects in the incoherent scattering of high energy particles, moving along classical trajectories in the averaged crystal potential, arises. Following Ref. [22], the multiple scattering theory in a homogeneous medium was initially applied [23] to sample the angles of classically moving particle scattering on the atomic planes. However, this approach could not be completely satisfactory, since the plane or string atomic density is strongly inhomogeneous, being characterized by the variation distance $u_{1}<0.1 \AA$, which makes it inadequate to use a fixed density value for impact parameters $b$ from the interval $u_{1} \leq b \leq R$, where $0.1 \AA<R \leq 0.5 \AA$ is the atom screening radius.

The influence of crystal atom density inhomogeneity on incoherent particle scattering had, in fact, already been known from the coherent bremsstrahlung theory [24], which predicted incoherent radiation and pair production reduction, caused by the same incoherent scattering. Being developed in plane wave [24] and reproduced in straightline trajectory approximations [2,3], the coherent bremsstrahlung theory remains valid at particle incident angles, at least a few times exceeding the critical channeling angle, involving only the incoherent scattering intensity averaged 
over the uniform particle flux implied by both plane wave and straight-line approximations.

However, at channeling and close to channeling conditions, particles spend different times at different locations in the plane of transverse motion or even do not reach some of them at all. The uniform flux approximation, accordingly, loses its applicability, making it necessary to describe incoherent particle scattering at each point individually, taking into consideration the behavior of the nuclear density at reaching $R>u_{1}$ distances from the trajectory. This problem is readily solved by the quantum treatment of transverse particle motion [25,26], being, however, both really necessary and practically feasible only at the electron and positron energies of a few dozen $\mathrm{MeV}$ and less. However, most of the current investigations are conducted at $\mathrm{GeV}[13,14,27]$ and higher [1-10,15-17,28-34] energies, at which the quantum description of particle motion in the averaged crystal field becomes both redundant and cumbersome, revealing the necessity for the introduction of classical particle motion features into the treatment of their incoherent scattering by the inhomogeneously distributed nuclei. Till now, a redefinition of the scattering impact parameter upper limit $R \rightarrow u_{1}[28-31,35]$ has been used, which did not take into consideration any incoherent scattering dependence on the transverse particle coordinate, giving, thus, a qualitative estimate only.

To develop a quantitative approach, the Wigner function of the transverse particle motion phase space [36] is applied for the local treatment of the incoherent scattering in Sec. III, in which an essentially novel formula for the local probability of incoherent scattering of a classically moving high energy particle is derived in the axial case. A method of consistent inclusion of the quantum scattering features into the simulations of relativistic particle classical motion in the averaged atomic string or plane potential is detailed in Sec. IV. We reveal that the strong enough inhomogeneity of the string atom nuclei distribution in the plane of transverse particle motion results in the impossibility to introduce a local scattering probability for the small angles and, to preserve the classical trajectory simulations, suggest to apply the newly introduced mean scattering angles. Considerable attention is also paid to the interrelation of single and multiple scattering processes, quite differently treated for decades.

\section{QUANTUM NATURE OF RELATIVISTIC PARTICLE SINGLE ATOM SCATTERING}

First of all, recall why, on the contrary to the coherent, the incoherent scattering becomes quantum at relativistic energies [11,19-21]. The inapplicability of classical mechanics to relativistic elementary (with a unit charge $|z|=1$ ) particle scattering by nuclei, in fact, directly follows from a comparison of the quantum (diffractional) angular uncertainty $\Delta \theta \sim \hbar / p b$, where $p$ is the particle momentum and $b$ the impact parameter, with the classical deflection angle

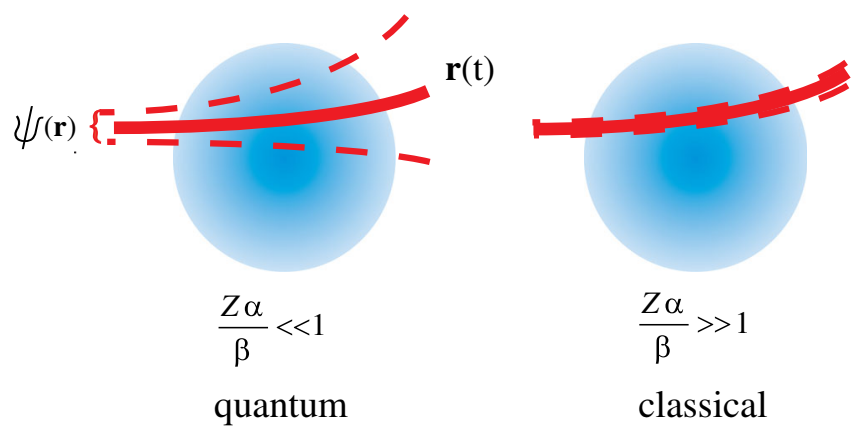

FIG. 1. Comparison of diffractive wave packet divergence $[\psi(\mathbf{r})$, dashed line] and classical particle deflection $[\mathbf{r}(\mathrm{t})$, solid line] in the quantum (left) and classical cases.

$\theta_{c l}=2 Z \alpha / p v b$, where $Z$ is the atomic number, $\alpha$ the fine structure constant, and $v=\beta c$ the particle velocity. Indeed, since $\Delta \theta \gg \theta_{c l}$ at $Z \alpha / \beta \ll 1$ and $\beta \simeq 1$, the scattering angle uncertainty exceeds the classical deflection angle, making the trajectory notion inapplicable for relativistic elementary particles, as Fig. 1 qualitatively illustrates. In other words, while the usage of classical trajectories in the averaged crystal potential becomes more and more justified with an energy increase (above several dozen $\mathrm{MeV}$ for electrons and positrons) [11], the classical binary collision method [18], on the opposite, becomes inapplicable. To illustrate the difference of quantum and classical predictions, let us compare the corresponding cross sections, evaluated for the Yukawa atomic potential, characterized by the adopted from Refs. [37,38] and used in GEANT4 screening radius $R=a_{T F}\left[1.13+3.76(\alpha Z / \beta)^{2}\right]^{-1 / 2}$, where $a_{T F}=$ $0.8853 a_{B} Z^{-1 / 3}$ and $a_{B}$ are, respectively, the ThomasFermi screening and Bohr radii. Figure 2 presents the angular dependence of the ratio of "quantum" to "classical" cross sections, the latter of which was evaluated following Ref. [39] in the relativistic case. These cross sections, as is well known, coincide at large transverse momentum transfers $q \gg \hbar / R$, corresponding to the scattering angles $\theta \gg \theta_{\min }=\hbar / p R$.

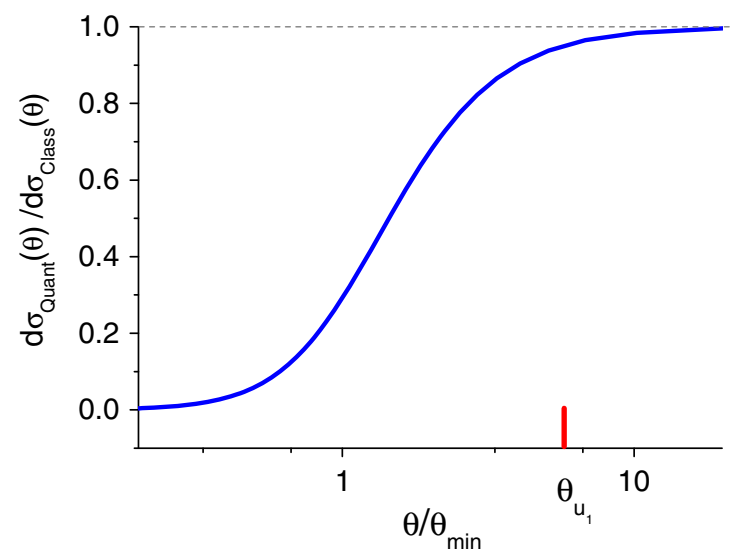

FIG. 2. The ratio of quantum to classical cross sections versus the scattering angle for a $\mathrm{Si}$ atom $(\alpha Z=0.102)$. 
However, at $q \leq \hbar / R$ or $\theta \leq \theta_{\min }$, when the screening effect becomes important, the classical approach overestimates [40] the scattering intensity, demonstrating the loss of its applicability. Note that both cross sections, compared in Fig. 2, have been calculated under the assumption of a uniform incident particle flux, for which classical mechanics overestimates the total cross section by the factor $(2 Z \alpha)^{-1}$, reaching 25 times for a $\mathrm{Si}$ atom. At the same time, opposite to the classical particle deflection in the binary collisions model, quantum mechanics cannot be directly applied to quantify the deflection of a particle moving along a classical trajectory.

It will be shown below that the correlations of particle collisions with string or plane atoms result in the incoherent scattering reduction similar, in a sense, to a screening at momentum transfers $q \leq \hbar / u_{1}$, corresponding to the scattering angles $\theta_{u_{1}} \leq \hbar / p u_{1}$, where $u_{1}$ is the root mean square amplitude of atom thermal vibrations. Since $\theta_{u_{1}}$ usually by $2-3$ times exceeds $\theta_{\min }$, one can expect $[25,35]$ (see also [28-31]) that collision correlations have to result in an incoherent scattering reduction, which will be numerically described below, taking for the first time into consideration its dependence on transverse coordinates.

To have a natural measure of the difference of incoherent scattering in crystals from the scattering in an amorphous medium, we will introduce a Kitagawa-Ohtsuki ansatz ("KO ansatz" below)

$$
\frac{d \Sigma_{K O}(\boldsymbol{\rho})}{d \boldsymbol{q}}=\frac{4 Z^{2} \alpha^{2} n_{n}(\rho)}{v^{2}\left[q^{2}+\kappa_{s c}^{2}\right]^{2}},
$$

inspired by the paper [22] of Kitagawa and Ohtsuki to be equal to the product of the unperturbed microscopic scattering cross section [relativistic cross section for the screened Coulomb (Yukawa) potential here] by the planar or, used below as an example, axial

$$
n_{n}(\rho)=\frac{\exp \left(-\rho^{2} / 2 u_{1}^{2}\right)}{2 \pi u_{1}^{2} d}
$$

nuclear number density, where $\rho$ is the distance from the atomic string symmetry axis (see Fig. 3) and $d$ interatomic distance in the string. One can mention that Eq. (1) resembles macroscopic cross sections, widely used in reactor physics, in which the nuclear number density can also vary widely.

However, KO ansatz (1) combines a classical particle coordinate $\rho$ with a quantum cross section only formally, neglecting the nuclear number density (2) variation, which

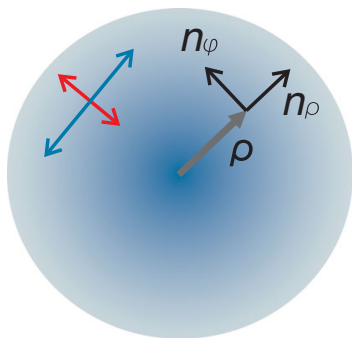

FIG. 3. Transverse radius-vector $\boldsymbol{\rho}$ and the directions $\boldsymbol{n}_{\boldsymbol{\rho}}, \boldsymbol{n}_{\boldsymbol{\varphi}}$ of extremal scattering intensities at the background of the string field. The double-sided arrows portray the excess of azimuthal scattering over the radial one.

exceeds $100 \%$ for the largest substantial impact parameters $b \sim R>u_{1}$, as well as missing both the effect of incoherent scattering reduction by correlations [25,28-31,35] and azimuthal asymmetry of the same [35]. To take the influence of the nuclear density inhomogeneity into consideration, we also introduce below some "macroscopic cross section," which, formally resembling the same in reactor physics, possesses, in a sense, a deeper meaning, combining in its general form the inseparable characteristics of the scattering probability and scatterers' density distribution. At the same time, in the high transfer momentum limit, the same macroscopic cross section will reduce to the product of nuclear density by a modified microscopic cross section, preserving the effect of the density (2) inhomogeneity and allowing one to incorporate quantum scattering effects in classical trajectory simulations.

\section{WIGNER FUNCTION APPLICATION TO SINGLE ATOM SCATTERING}

To introduce quantum features of incoherent scattering consistently into the classical picture of high energy particle motion in the averaged crystal potential, the Wigner function approach is most adequate [36]. Taking the axial case as an example, let us consider the Wigner function

$W(\boldsymbol{\rho}, \boldsymbol{q})=\frac{1}{\pi^{2}} \int \psi^{*}(\boldsymbol{\rho}+\chi) \psi(\boldsymbol{\rho}-\chi) \exp (2 i \boldsymbol{q} \chi) d^{2} \chi$,

determined in the two-dimensional phase space $(\boldsymbol{\rho}, \boldsymbol{q})$. $\boldsymbol{\rho}=(x, y), \boldsymbol{q}=\left(q_{x}, q_{y}\right)$, of the impact parameter plane nearly parallel to that of transverse motion of a particle, scattered by the residual atomic potential $[25,26]$

$$
\delta U_{a t}\left(\boldsymbol{\rho}-\boldsymbol{\rho}_{\boldsymbol{n}}, z\right)=U_{a t}\left(\boldsymbol{\rho}-\boldsymbol{\rho}_{\boldsymbol{n}}, z-z_{n}\right)-\int U_{a t}\left(\boldsymbol{\rho}-\boldsymbol{\rho}_{\boldsymbol{n}}, z-z_{n}\right) n_{n}\left(\rho_{n}\right) d^{2} \rho_{n} d z_{n},
$$

emerging after the substraction of the averaged one, already taken into consideration by the axial potential, which determines the classical particle motion. The potential (4) is small and localized enough to leave the three terms 


$$
\psi\left(\boldsymbol{\rho}-\boldsymbol{\rho}_{n}\right)=1-i \int_{a t} \delta U_{a t}\left(\boldsymbol{\rho}-\boldsymbol{\rho}_{n}, z\right) \frac{d z}{v}-\frac{1}{2}\left[\int_{a t} \delta U_{a t}\left(\boldsymbol{\rho}-\boldsymbol{\rho}_{n}, z\right) \frac{d z}{v}\right]^{2}
$$

of the eikonal wave function expansion, in which

$$
\int_{a t} \delta U_{a t}\left(\boldsymbol{\rho}-\boldsymbol{\rho}_{n}, z\right) \frac{d z}{v}=-\frac{i Z \alpha}{\pi v} \int \exp (i \boldsymbol{\rho} \boldsymbol{\rho}) \frac{\exp \left(-i \kappa \boldsymbol{\rho}_{n}\right)-\exp \left(-\kappa^{2} u_{1}^{2} / 2\right)}{\kappa^{2}+\kappa_{s}^{2}} d^{2} \kappa .
$$

Assuming a purely classical particle motion in the averaged potential both before and after the incoherent scattering at the point $\boldsymbol{\rho}$ in the transverse plane, we will evaluate the distribution in momentum $\boldsymbol{q}$, transferred to the particle at this point, being that of the catenation of the classical trajectories before and after the incoherent scattering. Substituting the wave function product

$$
\begin{aligned}
\psi^{*} & \left(\boldsymbol{\rho}+\chi-\boldsymbol{\rho}_{n}\right) \psi\left(\boldsymbol{\rho}-\chi-\boldsymbol{\rho}_{n}\right) \\
= & 1+i \int_{a t} \delta U_{a t}^{*}\left(\boldsymbol{\rho}+\chi-\boldsymbol{\rho}_{n}, z\right) \frac{d z}{v}-i \int_{a t} \delta U_{a t}\left(\boldsymbol{\rho}-\chi-\boldsymbol{\rho}_{n}, z\right) \frac{d z}{v}+\int_{a t} \delta U_{a t}^{*}\left(\boldsymbol{\rho}+\chi-\boldsymbol{\rho}_{n}, z\right) \frac{d z}{v} \int_{a t} \delta U_{a t}\left(\boldsymbol{\rho}-\chi-\boldsymbol{\rho}_{n}, z\right) \frac{d z}{v} \\
& -\frac{1}{2} \int_{a t} \delta U_{a t}\left(\boldsymbol{\rho}+\chi-\boldsymbol{\rho}_{n}, z\right) \frac{d z}{v} \int_{a t} \delta U_{a t}^{*}\left(\boldsymbol{\rho}+\chi-\boldsymbol{\rho}_{n}, z\right) \frac{d z}{v}-\frac{1}{2} \int_{a t} \delta U_{a t}^{*}\left(\boldsymbol{\rho}-\chi-\boldsymbol{\rho}_{n}, z\right) \frac{d z}{v} \int_{a t} \delta U_{a t}\left(\boldsymbol{\rho}-\chi-\boldsymbol{\rho}_{n}, z\right) \frac{d z}{v}
\end{aligned}
$$

into Eq. (3) and assuming that the unity in Eq. (7) corresponds to the particle propagation in the absence of incoherent scattering, one arrives to the "scattering" Wigner function

$$
\begin{aligned}
W_{\boldsymbol{\rho}_{n}}(\boldsymbol{\rho}, \boldsymbol{q})= & -\frac{8 Z \alpha}{\pi v}\left[\frac{\cos 2 \boldsymbol{q}\left(\boldsymbol{\rho}-\boldsymbol{\rho}_{n}\right)-\cos (2 \boldsymbol{q} \boldsymbol{\rho}) \exp \left(-2 q^{2} u_{1}^{2}\right)}{4 q^{2}+\kappa_{s}^{2}}\right]+\frac{4 Z^{2} \alpha^{2}}{\pi^{2} v^{2}} \int \exp (2 i \boldsymbol{\kappa} \boldsymbol{\rho})\left\{\frac{\exp \left[-i(\boldsymbol{q}+\boldsymbol{\kappa}) \boldsymbol{\rho}_{n}\right]-\exp \left[-(\boldsymbol{q}+\boldsymbol{\kappa})^{2} u_{1}^{2} / 2\right]}{(\boldsymbol{q}+\boldsymbol{\kappa})^{2}+\kappa_{s c}^{2}}\right\} \\
& \times\left\{\frac{\exp \left[i(\boldsymbol{q}-\boldsymbol{\kappa}) \boldsymbol{\rho}_{n}\right]-\exp \left[-(\boldsymbol{q}-\boldsymbol{\kappa})^{2} u_{1}^{2} / 2\right]}{(\boldsymbol{q}-\boldsymbol{\kappa})^{2}+\kappa_{s c}^{2}}\right\} d^{2} \kappa+\frac{4 Z^{2} \alpha^{2}}{\pi^{2} v^{2}} \exp (2 i \boldsymbol{q} \boldsymbol{\rho}) \int\left\{\frac{\exp \left[-i(\boldsymbol{q}+\boldsymbol{\kappa}) \boldsymbol{\rho}_{n}\right]-\exp \left[-(\boldsymbol{q}+\boldsymbol{\kappa})^{2} u_{1}^{2} / 2\right]}{(\boldsymbol{q}+\boldsymbol{\kappa})^{2}+\kappa_{s c}^{2}}\right\} \\
& \times\left\{\frac{\exp \left[-i(\boldsymbol{q}-\boldsymbol{\kappa}) \boldsymbol{\rho}_{n}\right]-\exp \left[-(\boldsymbol{q}-\boldsymbol{\kappa})^{2} u_{1}^{2} / 2\right]}{(\boldsymbol{q}-\boldsymbol{\kappa})^{2}+\kappa_{s c}^{2}}\right\} d^{2} \kappa,
\end{aligned}
$$

describing particle incoherent scattering at the point $\rho$ by an atom having a transverse radius vector $\boldsymbol{\rho}_{\boldsymbol{n}}$. In general, Eq. (8) can be applied to an arbitrary instant nuclear distribution in thermal vibration coordinates $\boldsymbol{\rho}_{n}$, as is discussed in Refs. [41,42]. However, a probabilistic interpretation of Eq. (8) does not look straightforward, since the leading, linear in $\alpha$, term strongly oscillates between positive and negative values. At the same time, the remaining quadratic terms in Eq. (8) reduce to the expected high momentum transfer limit of the relativistic Rutherford (Mott) cross section product by delta function $\delta\left(\boldsymbol{\rho}-\boldsymbol{\rho}_{\boldsymbol{n}}\right)$ at $q u_{1} \gg 1$.

To simplify the problem realistically, we choose here the traditional way $[24,25]$ of Eq. (8) convolution with the scattering nuclei distribution (2), which nullifies the linear in $\alpha$ contribution, leaving only the quadratic ones in Eq. (8). Owing to the Fourier integral presence in Eq. (6), the double integration in Eq. (3) results in the two-dimensional delta function, trivializing the double integration over $\kappa$ in one of the potentials (6), leaving the same in the another one the sole integral in the resulting expression:

$$
\begin{aligned}
\frac{d \Sigma(\boldsymbol{\rho})}{d \boldsymbol{q}}= & \frac{W(\boldsymbol{\rho}, \boldsymbol{q})}{d \boldsymbol{q}}=\frac{4 Z^{2} \alpha^{2}}{\pi^{2} v^{2}} \frac{1}{d}\left\{\int \cos (2 \boldsymbol{\kappa} \boldsymbol{\rho}) \frac{\exp \left(-2 \kappa^{2} u_{1}^{2}\right)-\exp \left[-\left(q^{2}+\kappa^{2}\right) u_{1}^{2}\right]}{\left[(\boldsymbol{q}+\boldsymbol{\kappa})^{2}+\kappa_{s c}^{2}\right]\left[(\boldsymbol{q}-\boldsymbol{\kappa})^{2}+\kappa_{s c}^{2}\right]} d^{2} \kappa\right. \\
& \left.+\cos (2 \boldsymbol{q} \boldsymbol{\rho})\left[\frac{\pi \ln \left(\sqrt{q^{2} / \kappa_{s c}^{2}+1}+q / \kappa_{s c}\right)}{q \sqrt{q^{2}+\kappa_{s c}^{2}}} \exp \left[-2 q^{2} u_{1}^{2}\right]-\int \frac{\exp \left[-\left(q^{2}+\kappa^{2}\right) u_{1}^{2}\right] d^{2} \kappa}{\left[(\boldsymbol{q}+\boldsymbol{\kappa})^{2}+\kappa_{s c}^{2}\right]\left[(\boldsymbol{q}-\boldsymbol{\kappa})^{2}+\kappa_{s c}^{2}\right]}\right]\right\}
\end{aligned}
$$

for the Wigner function. Representing the key result of the present paper, Eq. (9) will be used for the quantum treatment of incoherent scattering of a high energy particle, classically moving in the average crystal potential.

As will be shown in Sec. IV, Eq. (9) predicts quite peculiar behavior at momentum transfers $q<3 \hbar / u_{1}$, quickly approaching at $q>q_{a s}=3 \hbar / u_{1}$ its asymptote 


$$
\begin{aligned}
\frac{d \sigma_{\bmod }(\boldsymbol{\rho})}{d \boldsymbol{q}} & =\frac{1}{n_{n}(\boldsymbol{\rho})} \frac{d \Sigma(\boldsymbol{\rho})}{d \boldsymbol{q}} \\
& \simeq \frac{d \sigma_{\operatorname{Ruth}}(\boldsymbol{q})}{d \boldsymbol{q}}\left\{1-\frac{\rho^{2}}{2 q^{2} u_{1}^{4}}\left(1-8 e^{-\rho^{2} / 2 u_{1}^{2}-q^{2} u_{1}^{2}}\right)\left[\frac{2(\boldsymbol{q} \boldsymbol{\rho})^{2}}{q^{2} \rho^{2}}-1\right]-2 \pi^{2} \cos (2 \boldsymbol{q} \boldsymbol{\rho})\left[e^{-q^{2} u_{1}^{2}}-q^{2} u_{1}^{2} e^{-2 q^{2} u_{1}^{2}} \ln \frac{2 q}{\kappa_{s c}}\right] e^{\rho^{2} / 2 u_{1}^{2}}\right\}
\end{aligned}
$$

which can be used both to clarify the nature of the considered effects and to facilitate the simulations. At still larger momenta $q \gg \hbar / u_{1}$, corresponding to the impact parameters $b \sim \hbar / q \ll u_{1}$, at which the influence of the nuclear distribution inhomogeneity on the scattering process must vanish, Eq. (9) reduces to the product

$$
\left(\frac{d \Sigma(\boldsymbol{\rho})}{d \boldsymbol{q}}\right)_{q \gg \hbar / u_{1}} \rightarrow \frac{4 Z^{2} \alpha^{2} n_{n}(\rho)}{\pi^{2} q^{4}}=\frac{d \sigma_{\mathrm{Ruth}}}{d \boldsymbol{q}} n_{n}(\rho)
$$

of the Rutherford cross section by the nuclear density. Note that this "natural" limit takes place owing to both the residual incoherent scattering potential (4) introduction and averaging over the nuclei distribution (2).
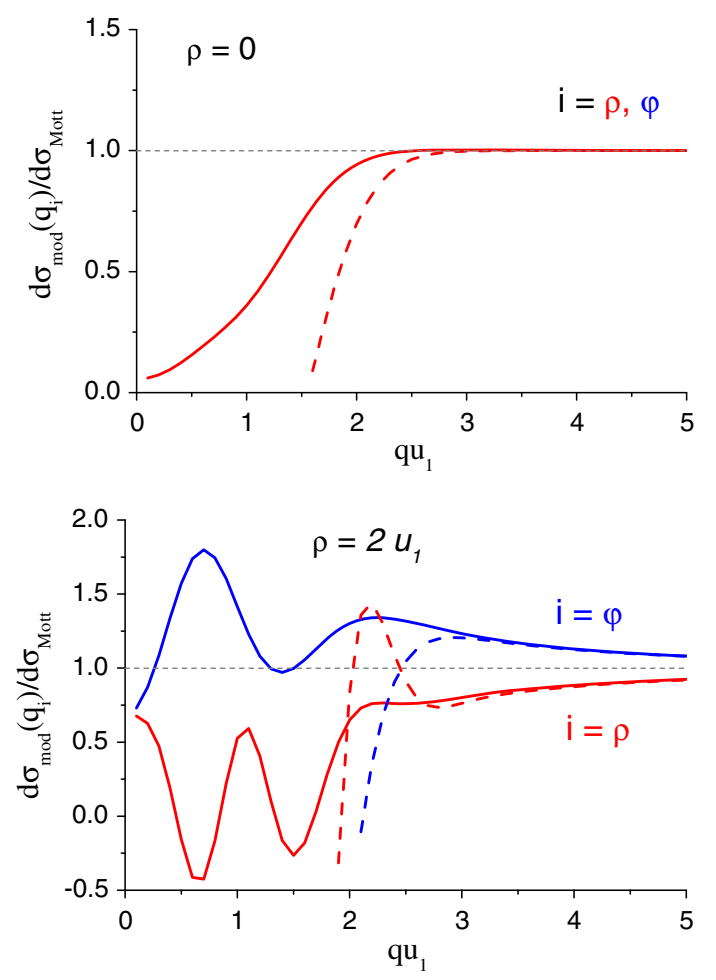

\section{QUANTUM INCOHERENT SCATTERING FEATURES AND THEIR INCORPORATION INTO CLASSICAL TRAJECTORY SIMULATIONS}

\section{A. Local scattering probability and mean square angles}

Still, both Eqs. (9) and (10) demonstrate a strong scattering asymmetry; we will consider Wigner function (9) properties for both radial $\boldsymbol{q}=\left(q_{\rho}, 0\right)$ and azimuthal $\boldsymbol{q}=$ $\left(0, q_{\varphi}\right)$ transferred momenta. Figure 4 , top, reveals that in the space region $\rho \leq u_{1}$ of the large, slowly changing nuclear density the Wigner function (9) is positive at any $q$ and can be used as a differential in momentum transfer scattering probability, determined at a definite transverse coordinate $\rho$ of the classical particle trajectory. Figure 4, top, also demonstrates that Eq. (9) for the first time locally describes the effect of incoherent scattering reduction by collision correlations $[24,25,28,31,35]$ at $q \sim \hbar / u_{1}$ in the $\rho \leq u_{1}$ region.
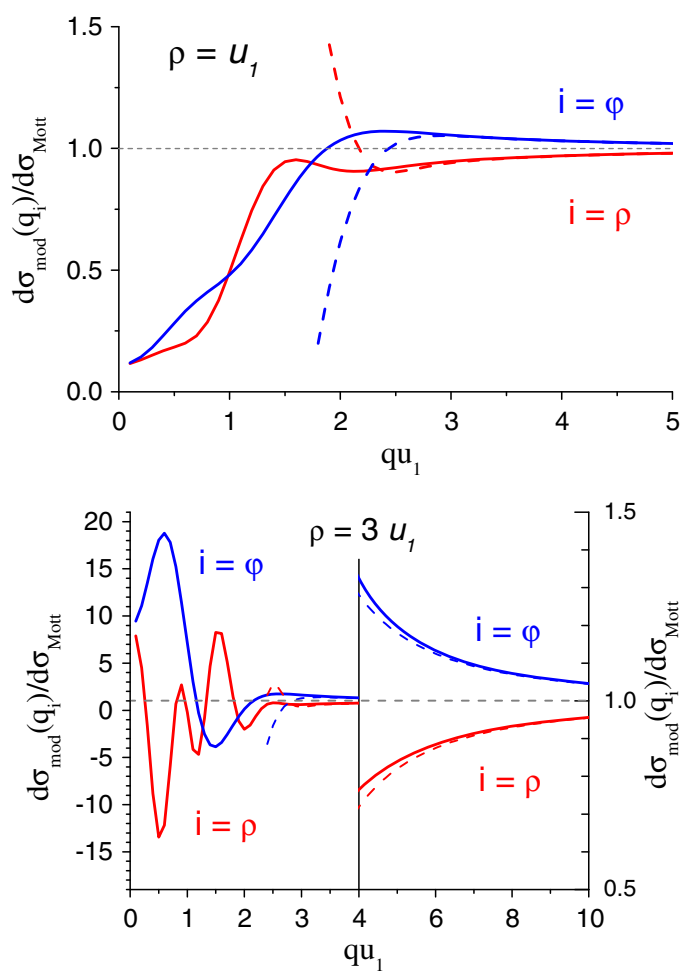

FIG. 4. Modified cross section dependence on the momentum transfer for radial $(i=\rho)$ and azimuthal $(i=\varphi)$ scattering directions, evaluated using the general (9) (solid lines) and asymptotic (10) (dashed lines) formulas for the radial coordinates $\rho=0$ (top left), $\rho=u_{1}$ (top right), $\rho=2 u_{1}$ (bottom left), and $\rho=3 u_{1}$ (bottom right) of the scattering point. 
At the same time, Fig. 4, bottom, concerning the region $2-3 u_{1}$ of both the low and strongly nonuniform nuclear density, reveals that the Wigner function can demonstrate more specific and pronounced quantum features, requiring an essentially new approach to incorporate them into classical particle trajectory simulations. Indeed, the asymptote (10) demonstrates that the Wigner function contains both the nuclear density proportional and disproportionate, cosine-dependent parts, describing, respectively, the local, high momentum transfer and the distant, interferencesensitive low-momentum one. The interference effects, naturally, induce Wigner function (9) oscillations at $\rho>a u_{1}$, where $a>1.5$, demonstrating the distant action of the region $\rho \sim a u_{1}-b$ of the high nuclear density $n_{n}\left(a u_{1}-b\right) \gg n_{n}\left(a u_{1}\right)$, becoming pronounced at collision parameters $u_{1} \leq b \leq R$. The same interference part of Eq. (9) also gives rise to the strong scattering asymmetry in the region $2-3 u_{1}$. Starting from the distance $\rho \simeq 1.68 u_{1}$, where nearly $25 \%$ of the string atom nuclei are located (see Fig. 5), the oscillating Wigner function (9) becomes negative for some $q$, making its direct probabilistic interpretation inconsistent.

A capability to attain negative values is an essential property of the Wigner function, reflecting its quantum nature. The Wigner function negativity does not allow one to treat it as a scattering probability for about $25 \%$ of the string nuclei (see Fig. 5). To retain, nevertheless, the simulation of quantum incoherent scattering of classically moving particles by most of the string nuclei, we put forward here a more indirect way, resembling the "multiple scattering approach," applied here, however, in the absence of positive scattering probability (cross section). The point is that, since the Rutherford cross section peaks at the lowest $q$, the small angle scattering process often manifests itself as a multiple one, characterized by the mean square scattering angle per unit length $[37,38,40]$. One can,

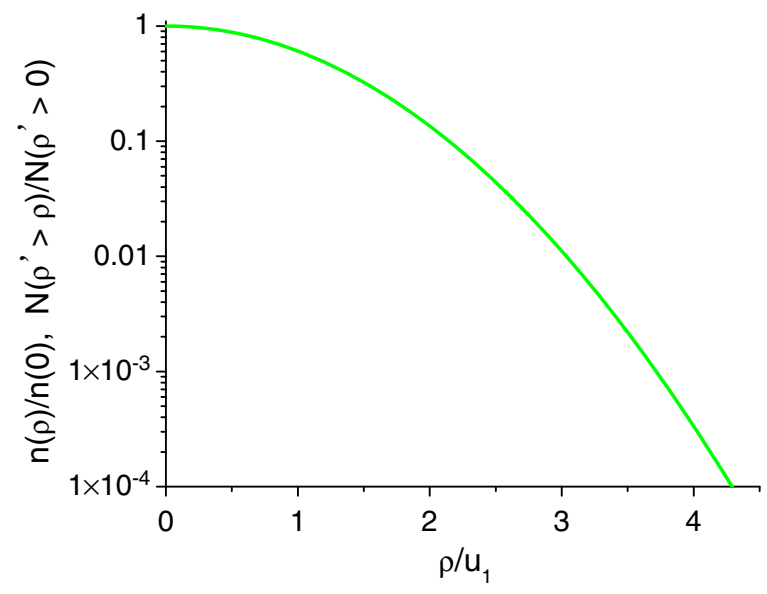

FIG. 5. Radial dependence of both the nuclear number density (2) and, proportional to the latter, number of the nuclei, situated at the radial distances $\rho^{\prime}>\rho$, exceeding the plotted $\rho$ value, both measured in units of their maximum values reached at $\rho=0$. similarly, use Eq. (9) to introduce the same for both radial $i=\rho$ and azimuthal $i=\varphi$ scattering directions:

$$
\frac{d \theta_{i}^{2}(\boldsymbol{\rho})}{d z}=\int_{q<q_{\max }^{\mathrm{MS}}} \frac{d \Sigma(\boldsymbol{\rho})}{d \boldsymbol{q}} \frac{q_{i}^{2}}{p^{2}} d^{2} q
$$

Figure 6 illustrates the dependence of the latter on the momentum integration limit $q_{\max }^{\mathrm{MS}}$, demonstrating again the drastic scattering asymmetry, which can be further taken into consideration by making $q_{\max }^{\mathrm{MS}}$ dependent on both the radial coordinate and the transferred momentum direction. Since the cross section approaches the Rutherford $q^{-4}$-type behavior at large $q$, for which the values (12) are definitely positive, one can expect that a large enough $q_{\max }^{\mathrm{MS}}$ value will assure (12) positivity, making possible routine scattering angle sampling. Suggesting here to use the positively determined mean square angles (12), we have in mind that, despite the absence of the local probabilistic interpretation, in general, the Wigner function is applicable to evaluate the consistent integral values of both the scattering probability and the mean square scattering angle, validating the suggested sampling method. Note also that the contribution of the large values of the Eq. (12) integrand, reached at small momentum transfers, most complicated by the distant quantum interference effects and related to the nuclear density inhomogeneity, is, in fact, strongly suppressed by the $q^{3}$ factor.

Comparing Fig. 4, bottom left, and Fig. 6, top right, both built for $\rho=2 u_{1}$, one can observe that, despite the negativity of the Wigner function, the mean square scattering angle can be positive at all $q_{\max }^{\mathrm{MS}}$. The same remains true up to $\rho \simeq 2.2 u_{1}$, for about $91 \%$ of the string nuclei (see Fig. 5), for which the value $q_{\max }^{\mathrm{MS}}$ is limited only by the requirement of Wigner function positivity.

However, the typical fall distance $\Delta \rho \simeq n_{n}(\rho) /\left|n_{n}^{\prime}(\rho)\right|=$ $u_{1}^{2} / \rho$ of the nuclear density (2) decreases well below $u_{1}$ at $>2 u_{1}$, extending the influence of nuclear inhomogeneity to the higher transferred momenta $q=\hbar / \Delta \rho>3 \hbar / u_{1}$. Indeed, for $\rho>2.5 u_{1}$, for about $5 \%$ of the string nuclei (see Fig. 5), $d \theta_{\rho}^{2}(\boldsymbol{\rho}) / d z$ becomes positive only somewhere in the region $q_{\max }^{\mathrm{MS}}>3 \hbar / u_{1}$ (Fig. 6, bottom), where the Wigner function is unambiguously positive and does not influence more on the $q_{\max }^{\mathrm{MS}}$ choice. At $\rho>3 u_{1}$, or for about $1 \%$ of the string nuclei, the region of negative $\theta_{\rho}^{2}$ extends even further to conclude that quantum effects become increasingly important for scattering by the small percentage of nuclei situated far enough from the atomic string, revealing the need for an alternative approach.

Note, that, though the nuclear number density (2) is rather low at $\rho>3 u_{1}$ (see Fig. 5), the extreme $\rho$ region is essential for the dechanneling of protons, positrons, and other positively charged particles, since the atom string nuclei take over the leading dechanneling role from atomic electrons right at $\rho \sim 2.5 u_{1}$. On the opposite, since, 

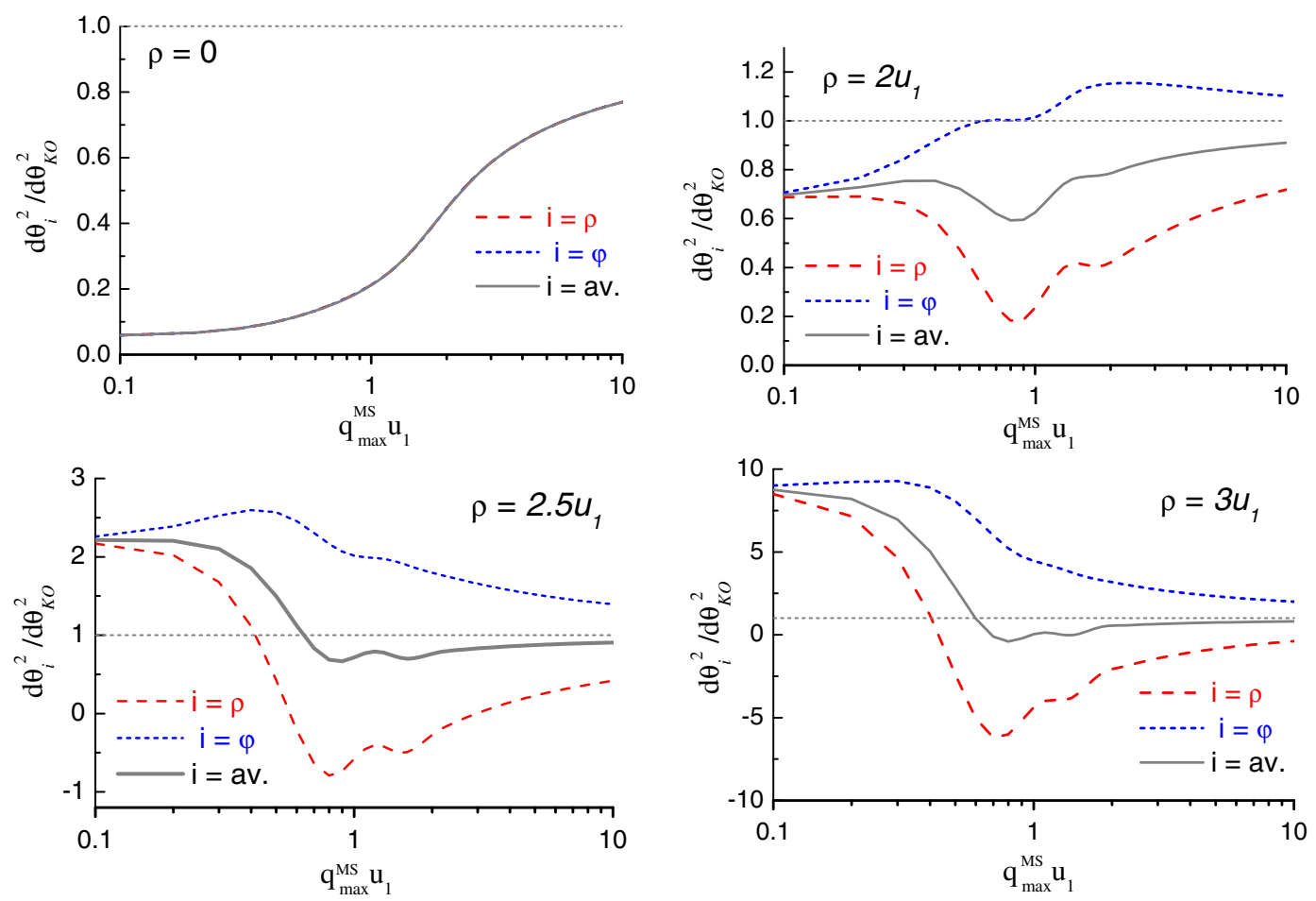

FIG. 6. Mean square angles of the scattering in both the radial $(i=\rho)$ and azimuthal $(i=\varphi)$ directions along with their average ( $i=a v$ ) versus the maximum contributing momentum transfer $q_{\max }^{M S}$ at $\rho=0$ (top left), $\rho=2 u_{1}$ (top right), $\rho=2.5 u_{1}$ (bottom left), and $\rho=3 u_{1}$ (bottom right), plotted as a ratio to the analogous positive values, evaluated using the KO ansatz (1).

normally, negatively charged particles regularly pass through the most dense regions, the quantum peculiarities of the scattering at $\rho \geq 3 u_{1}$ are hardly observable for them, and Eqs. (9), (10), and (12) describe the effect of incoherent scattering reduction practically completely.

\section{B. The necessity and relative role of single and multiple scattering in simulations}

Incoherent scattering treatment is crucial for both accuracy and efficiency of simulations of particle propagation through crystals. However, the really polar approaches of simulating all the incoherent channeling effects by using solely the mean square angle definition [22,23,43], on the one hand, and of sampling successive single scatterings either classically $[18,42]$ or quantumly [30,31], on the other, coexisted in the literature for decades.

Our approach [30], verified in Refs. [9,10,13,14,17,27, $34,44]$ and other investigations, includes the features of both of them, combining the sampling of both small angle multiple and large angle single scattering, the latter of which is most consonant to Ref. [31] in the necessity of its quantum treatment. The introduced Eq. (9) delivers both the firm grounds and calculation capabilities to the method [30]. However, before going to the latter, let us recall the peculiarities of the multiple Coulomb scattering theory $[37,38,45]$ application to the simulations of particle propagation in crystals, which are still under discussion.
Though the multiple Coulomb scattering theory $[37,38,45]$ (see also $[24,40,46,47]$ ) is thoroughly developed and widely tested experimentally, its formal application to the channeling simulations is highly questionable [30]. First of all, recall that the former predicts a nearly Gaussian angular distribution, characterized by the mean square angle

$\theta_{s}^{2}(l)=8 \pi n \frac{Z^{2} \alpha^{2}}{p^{2} v^{2}} \ln \left(\frac{\theta_{\max }}{\theta_{\min }}\right) l=16 \pi n \frac{Z^{2} \alpha^{2}}{p^{2} v^{2}} \ln \left(\frac{204}{Z^{1 / 3}}\right) l$,

where $n$ is a nuclear number density and $l$ a scattering length. We consider here only the ultrarelativistic small angle limit and adopt the slightly arbitrary numerical coefficients from Ref. [40]. Proceeding from quantum principles, the theory $[37,38,45]$ introduces both the lower

$$
\theta_{\min }=\frac{Z^{1 / 3}}{192} \frac{m}{p},
$$

where $m$ and $p$ are the particle mass and momentum, respectively, and the upper

$$
\theta_{\max }=\frac{274}{Z^{1 / 3}} \frac{m}{p}
$$



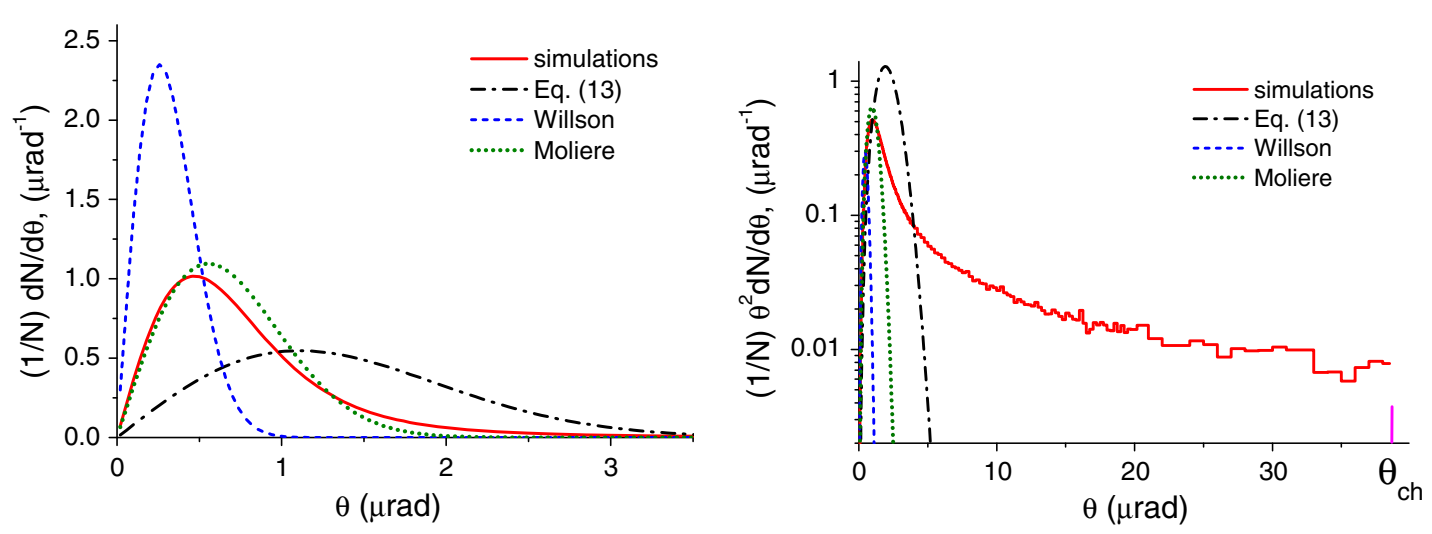

FIG. 7. Particle number (left) and angle square (right) distributions of $150 \mathrm{GeV}$ electrons, scattered within a typical trajectory simulation step (17): simulated directly (solid, red line) and evaluated using Gaussian polar angle distribution with mean square angles determined by the usual (13) (dash-dotted, black line), Williams's (dashed, blue line), and Moliere's (dotted, green lines) formulas.

scattering angle limits for high energy particles. The essential point of both Refs. [25,28,31,35] and the present paper is the widely discussed above need for a redefinition of the lower integration limit (14) in the presence of coherent scattering in crystals.

However, the upper one (15), being often formally used with both the mean square angle (13) and Gaussian distribution, is also mostly inapplicable to the trajectory simulations in crystals [30]. Instead of the coherent scattering effect at the small scattering angles, Eq. (13) inapplicability at the large ones is related with the unaccustomed short length of the trajectory simulation steps, the case of which finds quite specific and little-known complex treatment in the Moliere scattering theory [37,38,40,46] only. The latter elucidates that single scattering does not play a considerable role if only $2.5 \theta_{s}>\theta_{\max }$, as in a target with many radiation lengths thickness. However, if $2.5 \theta_{s}<\theta_{\max }$, the Gaussian distribution is applicable solely at the angles $\theta<2.5 \theta_{s}$, while at $2.5 \theta_{s}<\theta<\theta_{\max }$ single Coulomb scattering dominates.

The point is that the submicron length scale of simulated particle trajectories in crystals results in the root mean square angles $\theta_{s}$, which is drastically smaller than the maximal angle $\theta_{\max }(15)$, making both the single scattering essential and the application of the angle $\theta_{\max }$ as an upper limit in Eq. (13) highly inconsistent. Indeed, Eq. (13) involves a very wide single scattering angle interval $\theta_{s} \leq$ $\theta \leq \theta_{\max }$ into the evaluation of the width $\theta_{s}$ of the Gaussian distribution, covering, at the same time, only a much smaller interval $0<\theta<\theta_{s}$, which does include the angles $\theta_{s} \ll \theta \leq \theta_{\max }$, quite contradictory involved in $\theta_{s}$ evaluation through Eq. (15) at the same time. That is why, taken with the upper limit (15), Eq. (13) considerably overestimates the mean square angle, making the Gaussian unphysically wide-see Fig. 7. Reference [40] elucidates accordingly that at less than 200 collisions the true distribution, simulated here by an elemental Monte Carlo for Fig. 7, "is more sharply peaked at zero angle than a
Gaussian" [40,46], as one can indeed see in Fig. 7. That is why all the single scattering angles $\theta_{s} \leq \theta \leq \theta_{\max }$ should be disregarded in the mean scattering angle evaluation by redefining the upper limit in Eq. (15) according to the implicit condition $\theta_{\max } \simeq \theta_{s}\left(\theta_{\max }\right)[30,37,38]$.

The first realistic estimate [45]

$$
\theta_{c}=\frac{Z \alpha}{p v} \sqrt{4 \pi n l}
$$

of the actual width of the Gaussian distribution was introduced by equating the scattering probability at the angles $\theta>\theta_{c}$ to unity. However, the value (16), in fact, underestimates the angular distribution width (see Fig. 7), since a few scatterings by the angles $\theta<\theta_{c}$ often result in a multiple scattering angle $\theta>\theta_{c}$. The correct estimate $B \theta_{c}$, which contained a coefficient $B$, determined from some implicit condition, was finally introduced in Refs. [37,38] along with the involved formula for the true angular distribution.

To avoid the usage of the latter, we suggested [30] to sample both the small angle multiple and large angle single scattering jointly within each trajectory step. At that point, the minimal angle of single scattering can be chosen to be either equal or smaller than the root mean square multiple scattering angle, evaluated using an estimate of the same as the upper integration limit. This seemingly free choice of the latter is, in fact, validated by the adjustment of the single scattering process, self-adapting to the choice of the minimal single scattering angle. The multiple scattering simulation is, in fact, optional for this method, being used to avoid the simulation of some number of single scatterings. However, since the latter is quite modest for the short trajectory steps, the multiple scattering consideration can be reasonably abandoned in favor of the single scattering simulations with the properly chosen lower cutoff $[28,30,31]$. 
To compare the different mean square angle definitions, we have simulated the particle scattering in the screened Coulomb potential by the angles $0<\theta \leq \theta_{\max } \simeq 300 \mu \mathrm{rad}$ for the $150 \mathrm{GeV}$ energy used in the first experiment [48] on negatively charged particle multiple volume reflection [44]. Figure 7, left, presents the simulated angular distribution along with the three Gaussians, built for the mean square angles determined, respectively, by Eqs. (13)-(15), Eq. (16), and $\theta_{s}=B \theta_{c}$. All four distributions were evaluated for the typical trajectory step length

$$
\Delta l=0.02 \AA / \theta_{\mathrm{ch}} \sim 0.05 \mu \mathrm{m} .
$$

Figure 7, left, demonstrates, that, being applied with the upper limit (15), the definition (13) drastically overestimates the true width of the angular distribution, correctly predicted by the Moliere theory and reproduced by simulations.

At the same time, starting from $5 \mu \mathrm{rad}$, the Gaussian distribution with any mean square angle definition essentially underestimates the single scattering distribution tail, which both gives considerable contribution to the mean square angle up to the maximum single scattering angle $\theta_{\max } \simeq 300 \mu \mathrm{rad}$ and determines the actual, generally stochastic appearance of the scattering particle trajectories. Also, since $\theta_{\text {ch }} \sim 40 \mu \mathrm{rad} \gg 5 \mu \mathrm{rad}$, the single scattering process is completely responsible for the effects of instant dechanneling and rechanneling. At the same time, owing to both the fast cross section decrease with the angle and small trajectory step length, single scattering simulations are not time consuming, free from the problem with the logarithm in the mean square angle formula (13), and readily reproduce the not completely Gaussian small angle, the single scattering large angle, and the most difficult to handle intermediate angle regions of Moliere angular distribution, highly inappropriate for efficient sampling. For this reason, we have mostly relied in our simulations $[9,10,13,14,17,27,30,34,44]$ on the single scattering sampling, optionally supplemented with the multiple scattering one. The results (9)-(12) of the present paper make it possible to refine the simulation procedure, combining both single and multiple scattering processes most reasonably.

First of all, instead of the previously known average estimates, the effect of incoherent scattering reduction in crystals $[25,28,31,35]$ can now be treated quantitatively at any arbitrary point in the impact parameter plane. Being positive at any momentum transfer in the region $\rho<1.68 u_{1}$ of the moderately changing nuclear density, the Wigner function (9) can be consistently used as a scattering angle probability, determined on the classical trajectory. At that point, the coefficient of Rutherford cross section modification, depicted, in fact, in Fig. 4, top, can be applied to refine the single scattering angle sampling by the acceptance-rejection (von Neumann) method. The latter can be facilitated by using the asymptote (10) at $q>q_{a s}$ and further refined by introducing the momentum limit $q_{\max }$ dependence on both the radial coordinate and the transferred momentum direction. Also, both the multiple scattering model and mean square angles (12) can by optionally used for the small angles. However, this per se conventional approach remains consistent only when the Wigner function (9) is positive at all $q$, as in the discussed region $\rho<$ $1.68 u_{1}$ of about $75 \%$ of the string atom nuclei location.

The multiple scattering approach, applying the mean square angles (12), receives here the new fundamental justification of making it possible to simulate the incoherent scattering of a classically moving particle under the conditions when the Wigner function (9) does not reduce to the positively determined scattering probability (cross section), as in the region $\rho>1.68 u_{1}$ of a rapidly changing nuclear density. Indeed, when both the mean square angles (12) and Wigner function (9) are made positive by choosing a large enough integration limit $q_{\max }^{\mathrm{MS}}$, the former can be used to describe a contribution of the "small" momentum transfers $q<q_{\max }^{\mathrm{MS}}$ as a multiple scattering process, while either the latter or its asymptote (10) at $q>q_{a s}$ can be routinely treated as a scattering probability at the "large" ones $q_{\max }^{\mathrm{MS}}<q<$ $\theta_{\max } p$. At that point, the multiple scattering approach application at $\rho>1.68 u_{1}$ proves to be mandatory for the probabilistic incoherent scattering treatment on classical trajectories, opposite to multiple Coulomb scattering in an amorphous medium $[37,38,40,45,46]$, for which the positively determined cross section wittingly exists and multiple Coulomb scattering simulations are, as a matter of fact, used only to avoid the sampling of small single scatterings, the number of which usually exceeds hundreds of thousands in thick enough amorphous targets but, however, is really small for the trajectory steps in crystals. Since both the Wigner function and mean square angles attain negative values solely at $q<3 \hbar / u_{1}$ in the space region of $\rho<2.5 u_{1}$, the latter, in which more than $95 \%$ of the string atom nuclei are situated, should be classified at that, in which the present approach is most adequate.

The suggested method of the multiple scattering process introduction into simulations, which should be considered as a roughening reduction of the generally quantum incoherent scattering effects to the probabilistically treatable mean square angles, surely introduces some uncertainty, the scale of which can be estimated by comparing the value $q_{\max }^{\mathrm{MS}} / p$ with the typical angle of the considered problem, such as $\theta_{\mathrm{ch}}$, if channeling or close to potential barrier motion is considered. If the former angle is comparable with the latter, one should conclude that the precision of the suggested method of an incoherent scattering simulation of classically moving particles is limited, and, to improve it, an alternative approach is needed.

It is mostly expectable that the present method, making a strong emphasis on the precisely determined particle coordinate, loses both its relevance and precision at 
$\rho \geq 2.5 u_{1}$, where the nuclear density changes with $\rho$ much faster than the particle probability distribution in the transverse plane. An alternative approach, taking the latter situation into consideration, will be more adequate in the region $\rho \geq 3 u_{1}$ of a rather small and rapidly changing nuclear density, embracing about $1 \%$ of the nuclei. Being generally inessential for the negatively charged particles, the latter represents considerable interest for the "first principle" treatment of dechanneling of positively charged ones, which scatter on both nuclei and electrons with comparable intensities there.

The above consideration, undertaken here for the axial case, can be extended to the planar one either by a direct derivation, proceeding from Eqs. (3)-(8), or through the averaging of Eqs. (9)-(12) along straight trajectories traversing atomic strings. If we picture crystal planes as the ones being assembled from atomic strings, it becomes clear that the scattering attenuation in the radial direction, illustrated by both Figs. 4 and 6, has to result in the same nuclear dechanneling in the region of $2.5 u_{1} \sim 0.2 \AA$, critical for positively charged particle dechanneling.

Besides the numerous particle deflection problems, the developed approach can be applied to refine the radiation and pair production simulation method of Refs. [9,10,17,27,34].

\section{CONCLUSIONS}

A theory of incoherent particle scattering in oriented crystals in the high energy limit, in which relativistic particle motion in the averaged field of atomic strings and planes is mostly classical, is developed. Quantum effects of scattering by the atomic cores along a classical trajectory are treated by the Wigner function, determined in the phase space of transverse particle motion.

The effect of incoherent scattering reduction in the presence of atom distribution inhomogeneity is, for the first time, described on a classical particle trajectory by the local scattering probability identical to the Wigner function, being positively determined for most of the nuclei. At the same time, an impossibility to introduce the local scattering probability consistently for the rest of the latter is revealed, and essentially a novel definition of mean square scattering angles is introduced to incorporate the quantum scattering features into the classical trajectory simulations. A large azimuthal scattering asymmetry, which can considerably modify the positively charged particle nuclear dechanneling, is quantified. In general, the present theory makes it possible to refine a numerical treatment of any experiment on high energy particle scattering and radiation in crystals ever conducted or planned.

\section{ACKNOWLEDGMENTS}

The author is deeply obliged to the anonymous referee for both important corrections and improving suggestions.
He also acknowledges the fruitful 40-year discussions of the addressed problem with V.G. Baryshevsky, as well as with X. Artru, E. Bagli, V. N. Baier, L. Bandiera, A. O. Grubich, V. Guidi, S. B. Dabagov, I. Ya. Dubovskaya, I. D. Feranchuk, A. S. Lobko, V. A. Maisheev, A. Mazzolari, Y. H. Ohtsuki, M. I. Podgoretsky, N. F. Shulga, A. V. Solovyov, and A. I. Sytov.

[1] V. G. Baryshevsky, High-Energy Nuclear Optics of Polarized Particles (World Scientific, Singapore, 2012), .

[2] A. I. Akhiezer and N. F. Shul'ga, High Energy Electrodynamics in Matter (Gordon and Breach, New York, 1996).

[3] V. N. Baier, V. M. Katkov, and V. M. Strakhovenko, Electromagnetic Processes at High Energies in Oriented Single Crystals (World Scientific, Singapore, 1998).

[4] E. N. Tsyganov, Some aspects of the mechanism of a charged particle penetration through a monocrystal, Fermilab Report No. TM-682, 1976.

[5] V. M. Biryukov, Yu. A. Chesnokov, and V. I. Kotov, Crystal Channeling and Its Application at High-Energy Accelerators (Springer, Berlin, 1997).

[6] W. Scandale, Use of crystals for beam deflection in particle accelerators, Mod. Phys. Lett. A 27, 1230007 (2012).

[7] V. A. Baskov, V. A. Khablo, V. V. Kim, V. I. Sergienko, B. I. Luchkov, and V. Yu. Tugaenko, Electromagnetic showers in aligned crystals, Nucl. Instrum. Methods Phys. Res., Sect. B 122, 194 (1997).

[8] V. G. Baryshevskii and V. V. Tikhomirov, Synchrotrontype radiation processes in crystals and polarization phenomena accompanying them, Usp. Fiz. Nauk 159, 529 (1989) [Sov. Phys. Usp. 32, 1013 (1989)].

[9] L. Bandiera, E. Bagli, V. Guidi, A. Mazzolari, A. Berra, D. Lietti, M. Prest, E. Vallazza, D. De Salvador, and V. Tikhomirov, Broad and Intense Radiation Accompanying Multiple Volume Reflection of Ultrarelativistic Electrons in a Bent Crystal, Phys. Rev. Lett. 111, 255502 (2013).

[10] L. Bandiera, V. V. Tikhomirov, M. Romagnoni, N. Argiolas, E. Bagli, G. Ballerini, A. Berra, C. Brizzolani, R. Camattari, D. De Salvador, V. Haurylavets, V. Mascagna, A. Mazzolari, M. Prest, M. Soldani, A. Sytov, and E. Vallazza, Strong Reduction of the Effective Radiation Length in an Axially Oriented Scintillator Crystal, Phys. Rev. Lett. 121, 021603 (2018).

[11] J. Lindhard, Influence of crystal lattice on motion of energetic charged particles, Phys. Lett. 12, 126 (1964); Kgl. Dan. Vidensk. Selsk. Mat. Fys. Medd. 34, 1 (1965).

[12] M. W. Thompson, Effects of Proton Channeling at $2.8 \mathrm{MeV}$ of the $\mathrm{Cu}^{65}(p, n) \mathrm{Zh}^{65}$ Reaction Rate in a Single Crystal of Cu, Phys. Rev. Lett. 13, 756 (1964).

[13] A. Mazzolari, E. Bagli, L. Bandiera, V. Guidi, H. Backe, W. Lauth, V. Tikhomirov, A. Berra, D. Lietti, M. Prest, E. Vallazza, and D. De Salvador, Steering of a Sub-GeV Electron Beam through Planar Channeling Enhanced by Rechanneling, Phys. Rev. Lett. 112, 135503 (2014).

[14] A. I. Sytov, L. Bandiera1, D. De Salvador, A. Mazzolari1, E. Bagli1, A. Berra, S. Carturan, C. Durighello1, G. Germogli1, V. Guidi1, P. Klag, W. Lauth, G. Maggioni, M. Prest, M. Romagnoni, V. V. Tikhomirov, and 
E. Vallazza, Steering of sub-GeV electrons by ultrashort $\mathrm{Si}$ and Ge bent crystals, Eur. Phys. J. C 77, 901 (2017).

[15] A. V. Korol, A. V. Solov'yov, and W. Greiner, Channeling and Radiation in Periodically Bent Crystals, Springer Series on Atomic, Optical, and Plasma Physics, Vol. 69 (Springer-Verlag, Berlin, 2013).

[16] S. Bellucci and V. A. Maisheev, Radiation of relativistic particles for quasiperiodic motion in a transparent medium, J. Phys. Condens. Matter 18, S2083 (2006).

[17] V. G. Baryshevsky and V. V. Tikhomirov, Crystal undulators: From the prediction to the mature simulations, Nucl. Instrum. Methods Phys. Res., Sect. B 309, 30 (2013).

[18] M. T. Robinson and O. S. Oen, The channeling of energetic atoms in crystal lattices, Appl. Phys. Lett. 2, 30 (1963).

[19] N. Bohr, The penetration of atomic particles thorough matter, Kgl. Dan. Vidensk. Selsk. Mat. Fys. Medd. 18, 8 (1948).

[20] L. D. Landau and E. M. Lifshitz, Quantum Mechanics: Non-Relativistic Theory, 3rd ed. (Pergamon, New York, 1977), Vol. 3.

[21] P. Lervig, J. Lindhard, and V. Nielsen, Quantal treatment of directional effects for energetic charged particles in crystal lattices, Nucl. Phys. A96, 481 (1967).

[22] M. Kitagawa and Y. H. Ohtsuki, Modified dechanneling theory and diffusion coefficients, Phys. Rev. B 8, 3117 (1973).

[23] A. M. Taratin and S. A. Vorobev, Proton volume capture in channeling regime in bent crystal, Zh. Tekh. Fiz. 55, 1598 (1985) [Sov. Phys. Tech. Phys. 30, 927 (1985)].

[24] M. L. Ter-Mikaelian, High-Energy Electromagnetic Processes in Condensed Media (Wiley, New York, 1972).

[25] V. A. Bazylev and V. V. Goloviznin, Quantum theory of channeled electron and positron scattering in a crystal, Zh. Eksp. Teor. Fiz. 82, 1204 (1982) [Sov. Phys. JETP 55, 700 (1982)].

[26] V. A. Bazylev and S. B. Dabagov, Electromagnetic radiation under coherent and incoherent scattering of relativistic electrons in crystals, Zh. Tekh. Fiz. 58, 1563 (1988).

[27] L. Bandiera, E. Bagli, G. Germogli, V. Guidi, A. Mazzolari, H. Backe, W. Lauth, A. Berra, D. Lietti, M. Prest, D. De Salvador, E. Vallazza, and V. Tikhomirov, Investigation of the Electromagnetic Radiation Emitted by Sub-GeV Electrons in a Bent Crystal, Phys. Rev. Lett. 115, 025504 (2015).

[28] V. V. Tikhomirov, On the theory of electron-positron pair production in crystals, J. Phys. (Paris) 48, 1009 (1987).

[29] V. V. Tikhomirov, The position of the peak in the spectrum of $150 \mathrm{GeV}$ electron energy losses in a thin germanium crystal is proposed to be determined by radiation cooling, Phys. Lett. A 125, 411 (1987).

[30] V. V. Tikhomirov, Simulation of multi-GeV electron energy losses in crystals, Nucl. Instrum. Methods Phys. Res., Sect. B 36, 282 (1989).

[31] X. Artru, A simulation code for channeling radiation by ultrarelativistic electrons or positrons, Nucl. Instrum. Methods Phys. Res., Sect. B 48, 278 (1990).
[32] V. V. Tikhomirov, Quantitative theory of channeling particle diffusion in transverse energy in the presence of nuclear scattering and direct evaluation of dechanneling length, Eur. Phys. J. C 77, 483 (2017).

[33] V. G. Baryshevsky and V. V. Tikhomirov, The role of incoherent scattering in radiation processes at small angles of incidence of particles on crystallographic axes or planes, Zh. Eksp. Teor. Fiz. 90, 1908 (1986) [Sov. Phys. JETP 63, 1116 (1986)].

[34] V. Guidi, L. Bandiera, and V. Tikhomirov, Radiation generated by single and multiple volume reflection of ultrarelativistic electrons and positrons in bent crystals, Phys. Rev. A 86, 042903 (2012).

[35] V. L. Ljuboshits and M. I. Podgoretsky, Multiple Coulomb scattering of ultrarelativistic charged particles moving at small angles to crystallographic planes, Zh. Eksp. Teor. Fiz. 87, 717 (1984) [Sov. Phys. JETP 60, 409 (1984)].

[36] M. Ichikawa and Y. H. Ohtsuki, Inelastic-scattering theory for dechanneling, Phys. Rev. B 10, 1129 (1974).

[37] G. Moliére, Theorie der streuung schneller geladener teilchen II mehrfach- und vielfachstreuung 1, Z. Naturforschg. 3a, 78 (1948).

[38] H. A. Bethe, Moliere's theory of multiple scattering, Phys. Rev. 89, 1256 (1953).

[39] C. Lehmann and G. Leibfried, Higher order momentum approximations in classical collision theory, Z. Phys. 172, 465 (1963).

[40] J. D. Jackson, Classical Electrodynamics, 3rd ed. (Wiley, New York, 1999), ISBN 978-0-471-30932-1.

[41] X. Artru, Correlations in thermal vibrations of crystal atoms. Effect on dechanneling and bremsstrahlung, Nucl. Instrum. Methods Phys. Res., Sect. B 402, 21 (2017).

[42] I. A. Solovyov, A. V. Korol, and A. V. Solovyov, Multiscale Modeling of Complex Molecular Structure and Dynamics with MBN Explorer (Springer, New York, 2017).

[43] W. Scandale et al., Dechanneling of high energy particles in a long bent crystal, Nucl. Instrum. Methods Phys. Res., Sect. B 438, 38 (2019).

[44] V. V. Tikhomirov, Multiple volume reflection from different planes inside one bent crystal, Phys. Lett. A 655, 217 (2007).

[45] E. J. Williams, Multiple scattering of fast electrons and alpha-particles, and "curvature" of cloud tracks due to scattering, Phys. Rev. 58, 292 (1940).

[46] P. Sigmund and K. B. Winterborn, Small-angle multiple scattering of ions in the screening Coulomb region, Nucl. Instrum. Methods 119, 541 (1974).

[47] M. Tanabashi et al. (Particle Data Group), The review of particle physics (2018), Phys. Rev. D 98, 030001 (2018).

[48] W. Scandale et al., Observation of multiple volume reflection by different planes in one bent silicon crystal for high-energy negative particles, Europhys. Lett. 93, 56002 (2011). 